Supporting Information for:

\title{
Predicting Small Molecule Transfer Free Energies by Combining Molecular Dynamics Simulations and Deep Learning
}

W.F. Drew Bennett, ${ }^{* a}$ Stewart He, ${ }^{\mathrm{b}}$ Camille L. Bilodeau, ${ }^{\mathrm{a}}$ Derek Jones ${ }^{\mathrm{b}}$, Delin Sun, ${ }^{\mathrm{a}}$ Hyojin Kim, ${ }^{\mathrm{c}}$ Jonathan E. Allen, ${ }^{b}$ Felice C. Lightstone ${ }^{a}$ and Helgi I. Ingólfsson ${ }^{a}$

aBiochemical and Biophysical Systems Group, Biosciences and Biotechnology Division, Lawrence Livermore National Laboratory, 7000 East Avenue, Livermore, CA.

${ }^{\mathrm{b}}$ Global Security Computing Applications, Lawrence Livermore National Laboratory, 7000 East Avenue, Livermore, CA.

${ }^{\mathrm{c} C e n t e r}$ for Applied Scientific Computing, Lawrence Livermore National Laboratory, 7000 East Avenue, Livermore, CA.

*corresponding email bennett69@1lnl.gov 


\section{Methods}

\section{MD free energy calculations}

Molecule creation and parameterization: Small molecules were downloaded from the ZINC database ${ }^{1}$ for small drug-like molecules. Figure 1 shows the workflow that we used. From SMILES strings, molecules were built using RDKit. ${ }^{2}$ Parameters for the simulations were generated with FESetup, ${ }^{3}$ which is based on Antechamber from AMBER with AM1-BCC semiempirical partial charges. ${ }^{4}$ The generalizable AMBER force field parameters were used for the cyclohexane and the small molecules. ${ }^{5}$ Water was modelled with TIP3P. ${ }^{6}$ From 1500 base molecules that each contained a carboxylic acid, we replaced that functional group with 10 preselected functional groups $\left(-\mathrm{C}_{3} \mathrm{H}_{7},-\mathrm{SH},-\mathrm{NH} 2,-\mathrm{Cl},-\mathrm{F},-\mathrm{C}=0,-\mathrm{OH},-\mathrm{C}-\mathrm{OH},-\mathrm{C}(=\mathrm{O}-\mathrm{OH}),-\mathrm{S}(=\mathrm{O}=\mathrm{O}-\right.$ $\left.\mathrm{NH}_{2}\right)$ ).

MD simulation protocol: All simulations were run with GROMACS v5.1.4. ${ }^{7,8} \mathrm{~A} 2 \mathrm{fs}$ time step was used with stochastic integrator and a temperature of $298 \mathrm{~K}$. The box size was kept constant at a fixed volume, to maintain the interfacial tension. Long range electrostatics were calculated using the particle mesh Ewald method, ${ }^{9,10}$ with a real-space cut-off of $0.8 \mathrm{~nm}$. Lennard-Jones interactions were cut-off after $0.8 \mathrm{~nm}$, with a dispersion correction.

Umbrella Sampling: Each molecule was placed in the interface system, with water and cyclohexane, and its center of mass position in the direction normal to the interface was restrained with a harmonic potential. For one free energy profile, 13 simulations are run with the small molecule spaced in $0.2 \mathrm{~nm}$ increments, along the reaction coordinate, in the normal direction to the interface. To start each simulation, the molecule is placed at the correct location, in a random orientation and energy minimized conformation. The small molecule's Lennard-Jones and electrostatic interactions were slowly turned on, so the molecule slowly 'grows' into the equilibrated bulk solvent system. For each molecule's free energy profile, 13 simulations of $5 \mathrm{~ns}$ were run with a $1000 \mathrm{~kJ} / \mathrm{mol} \mathrm{nm}^{2}$ force constant. The weighted histogram analysis method was used to compute the free energy. ${ }^{11,12}$ Error was estimated using bootstrapping with 50 independent bootstraps, as implemented in g_wham. The average error for the umbrella sampling calculations was $3.3 \mathrm{~kJ} / \mathrm{mol}$ for the training data set, and 4.6 for the world approved drug set.

Thermodynamic Integration (TI): We used relative thermodynamic integration ${ }^{13}$ free energy calculations to expand our number of molecules for less computational cost compared to umbrella sampling. Each molecule was placed in a $\sim 4 \mathrm{~nm}^{3}$ box of water, cyclohexane, and the $4 \times 4 \times 8 \mathrm{~nm}$ interface system from the umbrella sampling simulations. A position restraint was used to keep the molecule at the interface (the trough in the free energy profiles). After energy minimization a 1 ns simulation of the drug in each environment was run using GROMACS. 7,8 Following the equilibration in each state, ' $\mathrm{fast}^{\text {' }} \mathrm{TI}$ calculations mutating $\mathrm{A}$ to $\mathrm{B}$ and $\mathrm{B}$ to $\mathrm{A}$ were run for $1 \mathrm{~ns}$ each. A similar method, using more replicates, but shorter simulations, has been recently used for amino acid mutation free energy calculations. ${ }^{14,15} \mathrm{Fig}$. S1 showing an example for the energy change during the perturbation. 
To compute relative free energies between the base molecule and adding one functional group was done using fast thermodynamic integration. Over one ns of simulation the $\lambda$ parameter that couples molecule $A$ and $B$, is changes from 0 to 1 , by $2 \times 10^{-6}$ increments (i.e. a $2 \mathrm{fs}$ time step). We also do the reverse transformation from 1 to 0 , starting from an equilibrated $B$ molecule transformation to $A$. We used stochastic dynamics to ensure numerical stability when atoms were being created/destroyed. These curves were numerically integrated using the trapezoidal method, giving the free energy change. The average free energy between 0-1 and 1-0 was then computed and reported. Error was estimated based on the difference between the two predictions and the mean. For all the mutations the average error in water was $1.18 \mathrm{~kJ} / \mathrm{mol}, 3.0 \mathrm{~kJ} / \mathrm{mol}$ at the interface, and $0.9 \mathrm{~kJ} / \mathrm{mol}$ in cyclohexane.

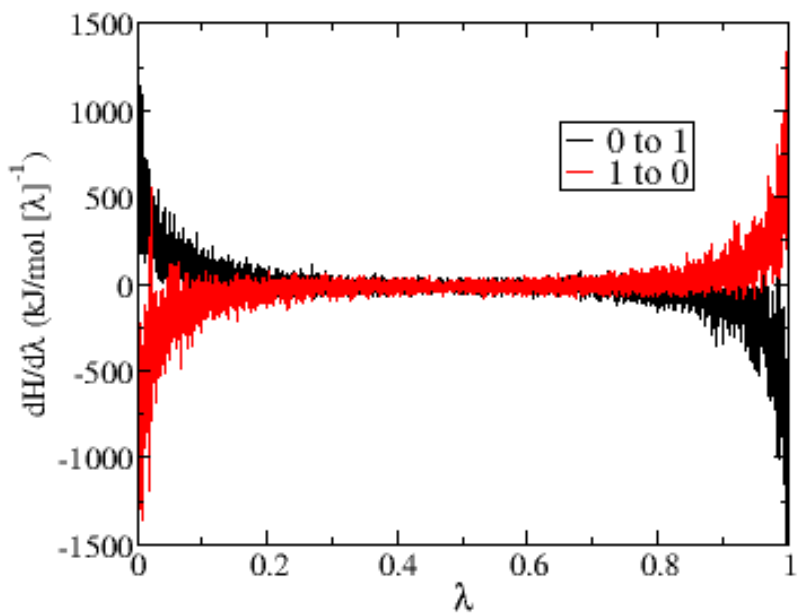

Figure S1: The forward and reverse transformation for mutating one small molecule to another using thermodynamic integration.

Analysis: By adding the free energy of the base molecule and the perturbed molecule in each environment, we can obtain the transfer free energies from all the relative $\mathrm{Tl}$ calculations. We calculated average molecular properties for each molecule in each environment, such as the molecules dipole, and number of hydrogen bonds, using GROMACS tools. For every atom in each molecule, we also counted the average number of water oxygen atoms and cyclohexane carbon atoms within $1 \mathrm{~nm}$. These cumulative radial distributions give information to the model regarding each atom's local chemical environment.

Atomic featurization: From the molecule's conformation at the end of the 1 ns equilibrium simulation, each atom is voxelized onto a 3D volume (voxel grid). Before calculating the voxels, we centered each molecule in a 3D volume, to ensure all the atoms were inside the grid. The volume region is split into $\mathrm{NxNxN}$ voxels, each of which is $0.5 \AA$ on each side, small enough so each voxel normally contains zero or one atom. Each voxel records 8 different feature types; is hydrogen, an atom that's not hydrogen, partial charge, Lennard-Jones sigma and epsilon, number of neighboring $\mathrm{O}$ atoms, number of neighboring $\mathrm{H}$ atoms, and number of neighboring $\mathrm{C}$ atoms. These features are calculated once for each of the 3 systems, water, hexane, and interface. We refer to partial charge, Lennard-Jones sigma, epsilon, and per voxel atom counts 
as a group as MD derived features. Models were trained with and without MD derived features and models trained with these features did better. It seems that, with 3DCNN and graph based networks, the choice of featurization still makes a difference. In the end each molecule is

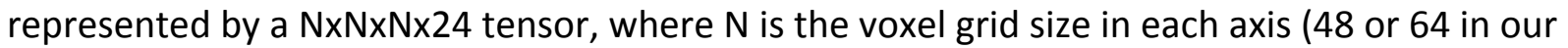
experiment).

We tested many different architectures, as an example we report the effect of voxel grid size, comparing $64 \times 64 \times 64\left(64^{\wedge} 3\right)$ to a voxel grid of $48 \times 48 \times 48\left(48^{\wedge} 3\right)$. Kuzminykh et al. ${ }^{16}$ tested the effect of different levels of Gaussian blur on the performance of autoencoders when processing 3D molecular structures. We have applied three levels of Gaussian blurs with $\sigma^{\prime} s$ of 1,2 , and 4 . Without a Gaussian blur each atom is assigned to a single voxel without collisions. As a result, less than $0.1 \%$ of voxels are populated with non-zero values. The first convolutional layer of our CNN uses $5 \times 5 \times 5$ kernels where local regions of $2.5 \AA$ are considered. The Gaussian blur increases the area of affect for each atom and the first layer is able to capture more information. For simplicity, we report only one model, as opposed to six (one for each $\sigma$ value and voxel grid size pair). The best $\sigma$ value and voxel size was chosen by looking at which $\sigma$ produced the best $R^{2}$ score on the validation dataset. This led to the decision to use $\sigma=4$ and a voxel grid size of $64 \times 64 \times 64$ for our final results. The results using other $\sigma$ values and voxel grid sizes were similar, but slightly lower.

The model is trained on the 15,000 molecules that were computed using atomistic MD. We tested training on both $\Delta \mathrm{G}_{\mathrm{W}-\mathrm{I}}$ and $\Delta \mathrm{G}_{\mathrm{W}-\mathrm{C}}$ (joined environment) and on only one at a time (single environment), for a multi-task learning approach. The data was split into training, validation, and testing at an 80:10:10 ratio. The split was performed using two different methods. The easier method, a random partitioning of the dataset, produced better results than using a scaffold split. This is expected because the training and testing distributions are more similar when using a random split. A scaffold split, as described in the main text, is a tougher experiment that tests the model's ability to generalize to a different distribution. Scaffold split results are reported in the main text.

Neural Network: The proposed convolutional neural network to model the 3D simulated data, similar to AtomNet, ${ }^{17}$ was implemented using Tensorflow. ${ }^{18}$ Figure 4 contains details on the choice of which layers and the size of each layer. We use ReLU non-linearities throughout and trained using an $20 \%$ dropout. We also include three molecule level features; number of hydrogen bonds, dipole, and radius of gyration (green in Fig. 4). These three molecule-wise features are concatenated with the activation of the last convolutional layer, which are fed into the first fully connected layer. Learning rate and the choice for $\sigma$ were the main hyperparameters. We used the Adam optimizer ${ }^{19}$ and the mean squared error cost function.

Spatial Graph Convolutional Neural Network: The SG-CNN was implemented using Pytorch and was similar to PotentialNet ${ }^{20}$. without non-covalent message passing. Each atom was represented using one-hot encoded atom types and the previously described MD-based parameters. Two message passing steps were used with a gather size of 128 . After the graph gather, the network was split into two fully connected neural networks with two hidden layers 


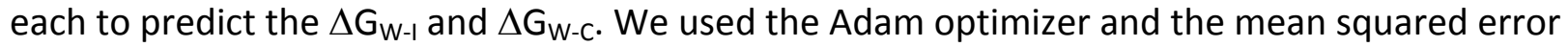
cost function.

Traditional Machine Learning: Each molecule's Morgan fingerprint ${ }^{21}$ was calculated in RDKit ${ }^{2}$ and folded into a 528 bit-vector (similar to the ECFP4 ${ }^{22}$ fingerprint). These chemical fingerprints were used to predict MD free energies using ElasticNet, ridge regression, and the random forest regressor implemented in the scikit-learn package. ${ }^{23}$

\section{Supporting Results}

We compared the free energy of transfer from water to cyclohexane to the free energy from water to the center of a POPC lipid bilayer. The free energy profiles were calculated using the same procedure as mentioned in the main text, but for the POPC 40 umbrella simulations were run, each for 200 ns to ensure convergence. These simulations were run using the AMBER14 lipid parameters ${ }^{24}$ and TIP3P for water ${ }^{6}$. GROMACS v5.1.47,8 was used with a $0.1 \mathrm{~nm}$ spacing between umbrella simulations and a $1000 \mathrm{~kJ} / \mathrm{mol} \mathrm{nm}^{2}$ force constant. The particle mesh Ewald method was used for long-range electrostatics ${ }^{9,10}$. Semi-isotropic pressure coupling was used with the Parrinello-Rahman barostat ${ }^{25}$, and a pressure of 1 bar.

Figure S2 shows the free energies for cyclohexanol and cyclohexane in a POPC bilayer compared to the water-cyclohexane system. For the cyclohexane, the free energy profile is similar for both POPC and water-cyclohexane. For cyclohexanol, the trough in the free energy at the interface is deeper for the water-cyclohexane system then in POPC.

\section{A. POPC system}

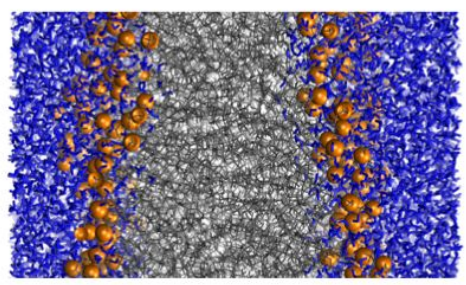

B. Water-cyclohexane system

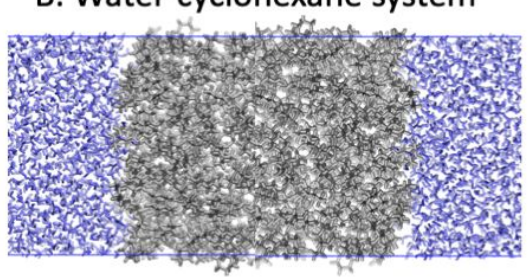

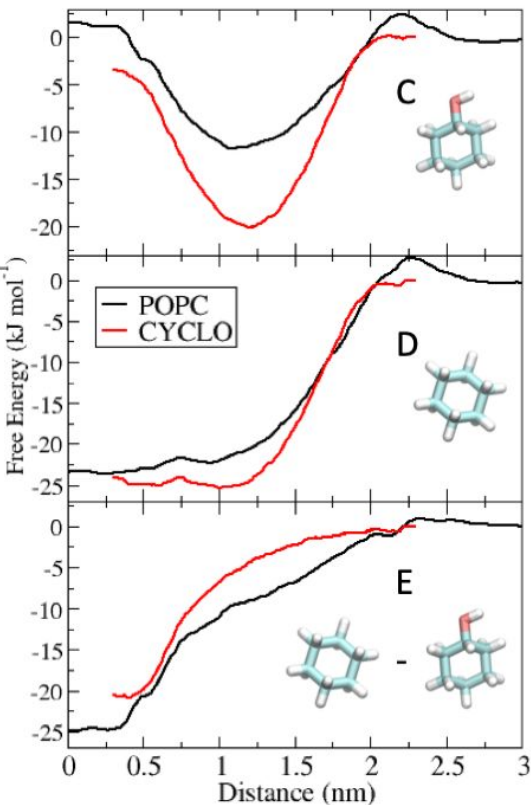

Figure S2: Free energy profiles for 2 small molecules in POPC compared to a water-cyclohexane interface. A. Snapshot of the POPC system, with water in blue and lipids grey with headgroups orange. B. Water-cyclohexane system, with water blue and cyclohexane grey. C. Free energy profiles for cyclohexanol, D. cyclohexane, E. the difference between the two molecules. 
Figure $\mathrm{S} 3$ shows the individual $\Delta \Delta \mathrm{G}_{\mathrm{W}-\mathrm{I}}$ and $\Delta \Delta \mathrm{G}_{\mathrm{W}-\mathrm{c}}$ for mutating each small molecule by one functional group. Each panel shows the functional group, with blue dots showing the range of free energies for each molecule. The larger red dot shows the average for all the molecules. Assuming a purely additivity for the free energy differences, then all these mutations should have a very similar free energy. The fact that such a large range exists, shows that simple additivity doesn't hold. The fact that the average values, match the expected chemical behaviour, suggests how additive models for computed log P (clogP) can be relatively successful. 

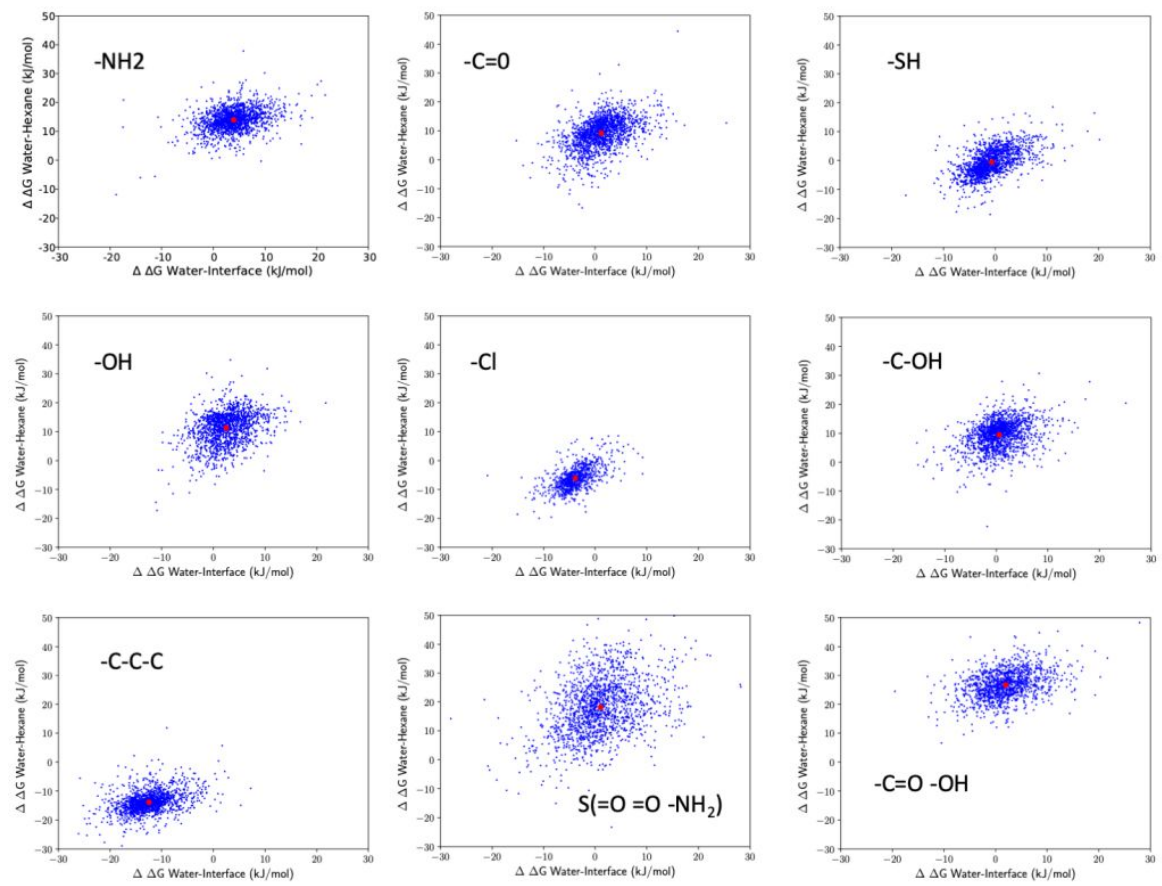

Figure S3: Relative free energies for mutating each small molecule by one functional group. Each panel shows the chemical modification. Blue dots show each free energy for waterinterface against water-cyclohexane. The red dot is the average for all the points for that modification.

Figure S4 shows the comparison for the Tanimoto distance for the random and scaffold splits between the test and validation sets and the world drug subset. This shows that scaffold split results in more diverse molecules, suggesting a more challenging test set for the model. In both cases the world drug set is distinct form the validation and other test set. This shows that training our model on the small molecule set and testing on the world drug set is a challenging task and illustrates the model's transferability. 

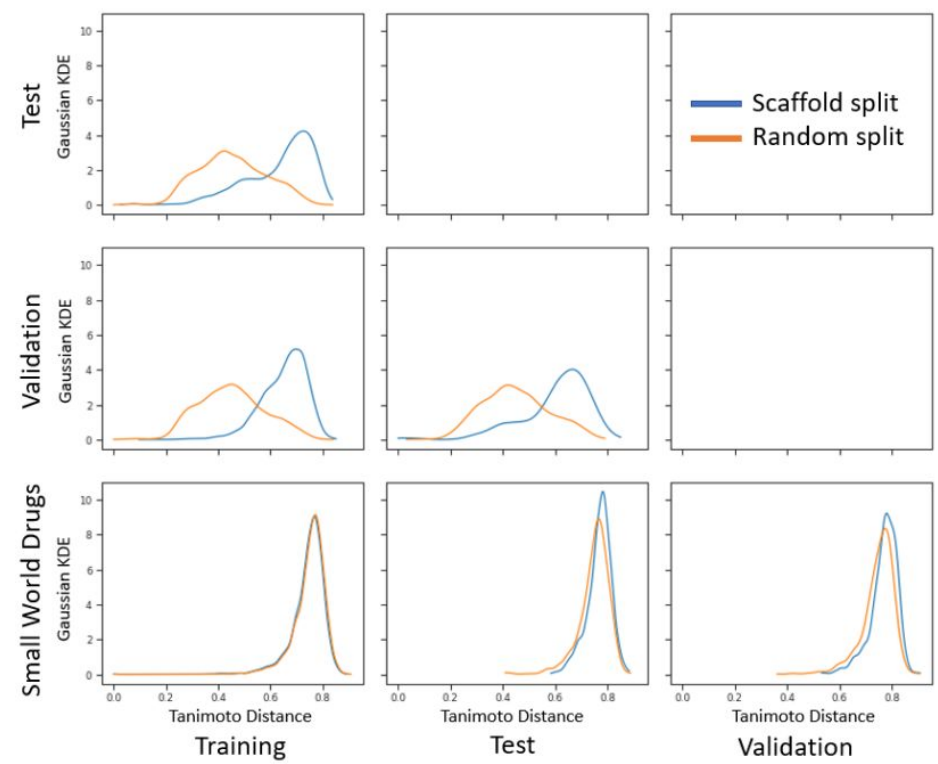

Figure S4: To measure the similarities between subsets of the data we calculated the Tanimoto distance between each sample in one subset to the nearest neighbor in the other subset. These distances were binned and a Gaussian KDE was fitted. Random split resulted in bell shaped curve centered around 0.4 . Scaffold splits resulted in distances around 0.7 . The small world drugs were significantly different from all data used in training with most distances in the 0.70.8 range.

Table S1-S4 show the results from various tests that were conducted as we optimized the model, testing the grid size, Gaussian blur level, scaffold split versus random split and various shallow learning methods. Fig. S5 shows scatter plots for the 3D-CNN predictions for the world drug test set. 
Table S1: ML results for predicting free energy with the $48 \times 48 \times 483 D-C N N$ using scaffold split or a random split.

\begin{tabular}{|c|c|c|c|c|}
\hline Model & RMSE & MAE & $\mathrm{R}^{2}$ & Pearson \\
\hline \multicolumn{5}{|c|}{ Scaffold Split $\Delta \mathrm{G}_{\mathrm{W}-1}$ results } \\
\hline $\begin{array}{l}\text { Single } \\
\text { env } \sigma=2\end{array}$ & 5.21 & 3.89 & 0.65 & 0.81 \\
\hline $\begin{array}{l}\text { Joined } \\
\text { env } \sigma=1\end{array}$ & 5.33 & 3.98 & 0.64 & 0.81 \\
\hline $\begin{array}{l}\text { Joined } \\
\text { env } \sigma=2\end{array}$ & 5.44 & 4.02 & 0.62 & 0.81 \\
\hline $\begin{array}{l}\text { Joined } \\
\text { env } \sigma=4\end{array}$ & 5.40 & 4.04 & 0.63 & 0.81 \\
\hline \multicolumn{5}{|c|}{ Scaffold Split $\Delta \mathrm{G}_{W-c}$ results } \\
\hline $\begin{array}{l}\text { Single } \\
\text { env } \sigma=4\end{array}$ & 7.32 & 5.49 & 0.83 & 0.91 \\
\hline $\begin{array}{l}\text { Joined } \\
\text { env } \sigma=1\end{array}$ & 7.53 & 5.68 & 0.82 & 0.91 \\
\hline $\begin{array}{l}\text { Joined } \\
\text { env } \sigma=2\end{array}$ & 7.53 & 5.65 & 0.82 & 0.91 \\
\hline $\begin{array}{l}\text { Joined } \\
\text { env } \sigma=4\end{array}$ & 7.60 & 5.70 & 0.82 & 0.91 \\
\hline \multicolumn{5}{|c|}{ Random Split: $\Delta G_{W-1}$ results } \\
\hline $\begin{array}{l}\text { Single } \\
\text { env } \sigma=4\end{array}$ & 4.63 & 3.50 & 0.72 & 0.85 \\
\hline $\begin{array}{l}\text { Joined } \\
\text { env } \sigma=1\end{array}$ & 4.79 & 3.62 & 0.70 & 0.84 \\
\hline $\begin{array}{l}\text { Joined } \\
\text { env } \sigma=2\end{array}$ & 4.72 & 3.54 & 0.71 & 0.84 \\
\hline $\begin{array}{l}\text { Joined } \\
\text { env } \sigma=4\end{array}$ & 4.77 & 3.59 & 0.70 & 0.85 \\
\hline \multicolumn{5}{|c|}{ Random Split: $\Delta G_{W-c}$ results } \\
\hline $\begin{array}{l}\text { Single } \\
\text { env } \sigma=4\end{array}$ & 6.62 & 5.02 & 0.86 & 0.93 \\
\hline $\begin{array}{l}\text { Joined } \\
\text { env } \sigma=1\end{array}$ & 6.68 & 5.06 & 0.86 & 0.93 \\
\hline $\begin{array}{l}\text { Joined } \\
\text { env } \sigma=2\end{array}$ & 6.56 & 4.94 & 0.87 & 0.93 \\
\hline $\begin{array}{l}\text { Joined } \\
\text { env } \sigma=4\end{array}$ & 6.72 & 5.12 & 0.86 & 0.93 \\
\hline
\end{tabular}


Table S2: ML Results for predicting free energy with the $64 \times 64 \times 64$ 3D-CNN using scaffold split or a random split.

\begin{tabular}{|c|c|c|c|c|}
\hline Model & RMSE & MAE & $\mathrm{R}^{2}$ & Pearson \\
\hline \multicolumn{5}{|c|}{ Scaffold Split: $\Delta G_{W-1}$ results } \\
\hline $\begin{array}{l}\text { Single env } \\
\sigma=4\end{array}$ & 5.23 & 3.90 & 0.65 & 0.81 \\
\hline $\begin{array}{l}\text { Joined env } \\
\sigma=1\end{array}$ & 5.48 & 4.08 & 0.62 & 0.80 \\
\hline $\begin{array}{l}\text { Joined env } \\
\sigma=2\end{array}$ & 5.34 & 4.02 & 0.64 & 0.81 \\
\hline $\begin{array}{l}\text { Joined env } \\
\sigma=4\end{array}$ & 5.29 & 3.91 & 0.64 & 0.81 \\
\hline \multicolumn{5}{|c|}{ Scaffold Split: $\Delta G_{W-c}$ results } \\
\hline $\begin{array}{l}\text { Single env } \\
\sigma=4\end{array}$ & 7.41 & 5.56 & 0.83 & 0.91 \\
\hline $\begin{array}{l}\text { Joined env } \\
\sigma=1\end{array}$ & 7.56 & 5.69 & 0.82 & 0.91 \\
\hline $\begin{array}{l}\text { Joined env } \\
\sigma=2\end{array}$ & 7.60 & 5.72 & 0.82 & 0.91 \\
\hline $\begin{array}{l}\text { Joined env } \\
\sigma=4\end{array}$ & 7.54 & 5.67 & 0.82 & 0.91 \\
\hline \multicolumn{5}{|c|}{ Random Split: $\Delta G_{W-1}$ results } \\
\hline $\begin{array}{l}\text { Single env } \\
\sigma=4\end{array}$ & 4.67 & 3.51 & 0.71 & 0.85 \\
\hline $\begin{array}{l}\text { Joined env } \\
\sigma=1\end{array}$ & 4.81 & 3.63 & 0.70 & 0.84 \\
\hline $\begin{array}{l}\text { Joined env } \\
\sigma=2\end{array}$ & 4.86 & 3.62 & 0.69 & 0.84 \\
\hline $\begin{array}{l}\text { Joined env } \\
\sigma=4\end{array}$ & 4.62 & 3.49 & 0.72 & 0.85 \\
\hline \multicolumn{5}{|c|}{ Random Split: $\Delta G_{W-c}$ results } \\
\hline $\begin{array}{l}\text { Single env } \\
\sigma=4\end{array}$ & 6.67 & 5.08 & 0.86 & 0.93 \\
\hline $\begin{array}{l}\text { Joined env } \\
\sigma=1\end{array}$ & 6.65 & 5.02 & 0.86 & 0.93 \\
\hline $\begin{array}{l}\text { Joined env } \\
\sigma=2\end{array}$ & 6.73 & 5.11 & 0.86 & 0.93 \\
\hline $\begin{array}{l}\text { Joined env } \\
\sigma=4\end{array}$ & 6.56 & 5.01 & 0.87 & 0.93 \\
\hline
\end{tabular}


Table S3: ML prediction results for small drugs from the world approved drugs dataset.

\begin{tabular}{|c|c|c|c|c|}
\hline Model & RMSE & MAE & $\mathrm{R}^{2}$ & Pearson \\
\hline \multicolumn{5}{|c|}{ Random Split: $\Delta G_{W-I}$ results } \\
\hline $\begin{array}{l}\text { Joined env } 48 \\
\text { voxels } \sigma=2\end{array}$ & 4.78 & 3.66 & 0.81 & 0.92 \\
\hline $\begin{array}{l}\text { Joined env } 64 \\
\text { voxels } \sigma=4\end{array}$ & 5.29 & 4.03 & 0.77 & 0.90 \\
\hline \multicolumn{5}{|c|}{ Random Split: $\Delta \mathrm{G}_{\mathrm{W}-\mathrm{c}}$ results } \\
\hline $\begin{array}{l}\text { Joined env } 48 \\
\text { voxels } \sigma=2\end{array}$ & 13.31 & 8.81 & 0.70 & 0.84 \\
\hline $\begin{array}{l}\text { Joined env } 64 \\
\text { voxels } \sigma=4\end{array}$ & 14.88 & 9.66 & 0.62 & 0.80 \\
\hline \multicolumn{5}{|c|}{ Scaffold Split: $\Delta \mathrm{G}_{\mathrm{W}-\mathrm{I}}$ results } \\
\hline Model & RMSE & MAE & $\mathrm{R}^{2}$ & Pearson \\
\hline $\begin{array}{l}\text { Joined env } 48 \\
\text { voxels } \sigma=2\end{array}$ & 5.36 & 4.09 & 0.77 & 0.91 \\
\hline $\begin{array}{l}\text { Joined env } 64 \\
\text { voxels } \sigma=4\end{array}$ & 5.73 & 4.28 & 0.73 & 0.88 \\
\hline \multicolumn{5}{|c|}{ Scaffold Split: $\Delta G_{W-c}$ results } \\
\hline $\begin{array}{l}\text { Joined env } 48 \\
\text { voxels } \sigma=1\end{array}$ & 14.20 & 9.00 & 0.65 & 0.81 \\
\hline $\begin{array}{l}\text { Joined env } 64 \\
\text { voxels } \sigma=4\end{array}$ & 15.33 & 9.73 & 0.60 & 0.78 \\
\hline
\end{tabular}

Table S4: Machine learning results for predicting the $\Delta \mathrm{G}_{\mathrm{W}-\mathrm{c}}$ and $\Delta \mathrm{G}_{\mathrm{W}-\mathrm{I}}$ with ECFP4 fingerprints.

\begin{tabular}{lccll}
\hline \multicolumn{1}{c}{ Model } & RMSE & MAE & $\mathrm{R}^{2}$ & Pearson \\
\hline$\Delta \mathrm{G}_{\mathrm{W}-\mathrm{I}}$ results & & & & \\
\hline $\begin{array}{l}\text { ElasticNet } \\
\text { Ridge }\end{array}$ & 5.44 & 4.22 & 0.47 & $\mathbf{0 . 7 5}$ \\
$\begin{array}{l}\text { regression } \\
\text { Random }\end{array}$ & $\mathbf{5 . 3 5}$ & $\mathbf{4 . 1 0}$ & 0.49 & 0.74 \\
\begin{tabular}{l} 
Forest \\
\hline $\mathrm{G}_{\mathrm{W}-\mathrm{c} \text { results }}$
\end{tabular} & 5.66 & 4.38 & $\mathbf{0 . 4 3}$ & 0.74 \\
\hline $\begin{array}{l}\text { ElasticNet } \\
\text { Ridge } \\
\text { regression }\end{array}$ & $\mathbf{8 . 8 8}$ & 6.61 & 0.74 & 0.87 \\
$\begin{array}{l}\text { Random } \\
\text { Forest }\end{array}$ & $\mathbf{8 . 4 1}$ & $\mathbf{6 . 0 0}$ & $\mathbf{0 . 7 7}$ & $\mathbf{0 . 8 8}$
\end{tabular}




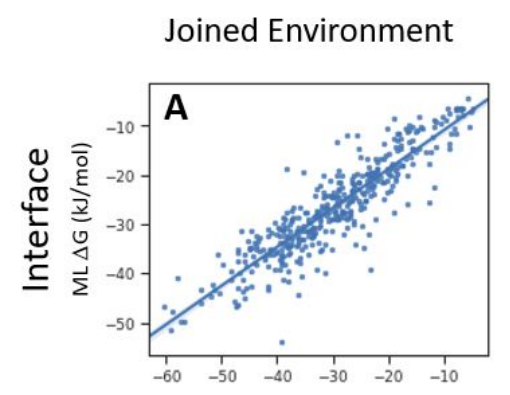

\section{Single Environment}
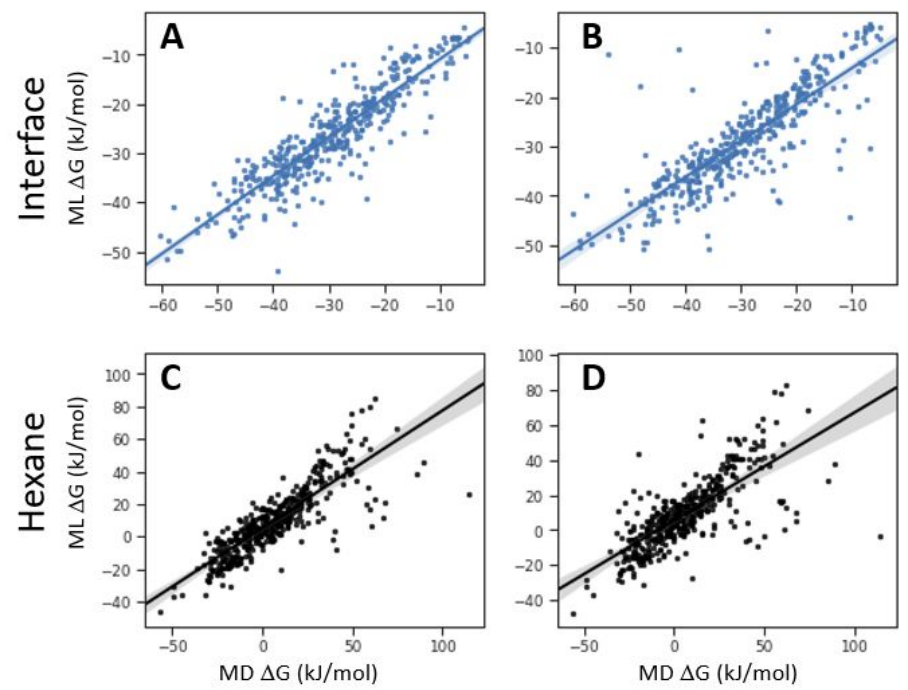

Figure S5: Results from the 3D-CNN based for the world approved drugs subset from the ML model trained on the small molecule dataset. A. Interface trained on both. B. Interface trained only on interface. C. Cyclohexane from both models. D. Cyclohexane only model. The lines indicate the best fit on each scatter plot. 


\section{References}

(1) Irwin, J. J.; Sterling, T.; Mysinger, M. M.; Bolstad, E. S.; Coleman, R. G. ZINC: A Free Tool to Discover Chemistry for Biology. Journal of chemical information and modeling 2012, 52, 1757-1768.

(2) Landrum, G. RDKit: Open-source cheminformatics (accessed Jan 1, 2019).

(3) Loeffler, H. H.; Michel, J.; Woods, C. FESetup: Automating Setup for Alchemical Free Energy Simulations. Journal of Chemical Information and Modeling 2015, 55, 2485-2490.

(4) Jakalian, A.; Jack, D. B.; Bayly, C. I. Fast, Efficient Generation of High-quality Atomic Charges. AM1-BCC Model: II. Parameterization and Validation. Journal of computational chemistry 2002, 23, 1623-1641.

(5) Wang, J.; Wolf, R. M.; Caldwell, J. W.; Kollman, P. A.; Case, D. A. Development and Testing of a General Amber Force Field. J Comput Chem 2004, 25, 1157-1174. https://doi.org/10.1002/jcc.20035.

(6) Jorgensen, W. L.; Chandrasekhar, J.; Madura, J. D.; Impey, R. W.; Klein, M. L. Comparison of Simple Potential Functions for Simulating Liquid Water. J. Chem. Phys. 1983, 79, 926935. https://doi.org/10.1063/1.445869.

(7) Abraham, M. J.; Murtola, T.; Schulz, R.; Páll, S.; Smith, J. C.; Hess, B.; Lindahl, E. GROMACS: High Performance Molecular Simulations through Multi-Level Parallelism from Laptops to Supercomputers. SoftwareX 2015, 1-2, 19-25.

https://doi.org/10.1016/j.softx.2015.06.001.

(8) Van Der Spoel, D.; Lindahl, E.; Hess, B.; Groenhof, G.; Mark, A. E.; Berendsen, H. J. GROMACS: Fast, Flexible, and Free. Journal of computational chemistry 2005, 26, 17011718.

(9) Darden, T.; York, D.; Pedersen, L. Particle Mesh Ewald: An N· Log (N) Method for Ewald Sums in Large Systems. The Journal of chemical physics 1993, 98, 10089-10092.

(10) Essmann, U.; Perera, L.; Berkowitz, M. L.; Darden, T.; Lee, H.; Pedersen, L. G. A Smooth Particle Mesh Ewald Method. The Journal of chemical physics 1995, 103, 8577-8593.

(11) Kumar, S.; Rosenberg, J. M.; Bouzida, D.; Swendsen, R. H.; Kollman, P. A. The Weighted Histogram Analysis Method for Free-energy Calculations on Biomolecules. I. The Method. Journal of computational chemistry 1992, 13, 1011-1021.

(12) Hub, J. S.; De Groot, B. L.; Van Der Spoel, D. G_wham? A Free Weighted Histogram Analysis Implementation Including Robust Error and Autocorrelation Estimates. Journal of chemical theory and computation 2010, 6, 3713-3720.

(13) Kirkwood, J. G. Statistical Mechanics of Fluid Mixtures. The Journal of chemical physics 1935, 3, 300-313.

(14) Gapsys, V.; Michielssens, S.; Seeliger, D.; de Groot, B. L. Accurate and Rigorous Prediction of the Changes in Protein Free Energies in a Large-Scale Mutation Scan. Angewandte Chemie 2016, 128, 7490-7494.

(15) Gapsys, V.; Michielssens, S.; Seeliger, D.; de Groot, B. L. Pmx: Automated Protein Structure and Topology Generation for Alchemical Perturbations. Journal of computational chemistry 2015, 36, 348-354.

(16) Kuzminykh, D.; Polykovskiy, D.; Kadurin, A.; Zhebrak, A.; Baskov, I.; Nikolenko, S.; Shayakhmetov, R.; Zhavoronkov, A. 3D Molecular Representations Based on the Wave 
Transform for Convolutional Neural Networks. Mol. Pharmaceutics 2018, 15, 4378-4385. https://doi.org/10.1021/acs.molpharmaceut.7b01134.

(17) Wallach, I.; Dzamba, M.; Heifets, A. AtomNet: A Deep Convolutional Neural Network for Bioactivity Prediction in Structure-Based Drug Discovery. arXiv preprint arXiv:1510.02855 2015.

(18) Martín Abadi; Ashish Agarwal; Paul Barham; Eugene Brevdo; Zhifeng Chen; Craig Citro; Greg S. Corrado; Andy Davis; Jeffrey Dean; Matthieu Devin; Sanjay Ghemawat; Ian Goodfellow; Andrew Harp; Geoffrey Irving; Michael Isard; Jia, Y.; Rafal Jozefowicz; Lukasz Kaiser; Manjunath Kudlur; Josh Levenberg; Dandelion Mané; Rajat Monga; Sherry Moore; Derek Murray; Chris Olah; Mike Schuster; Jonathon Shlens; Benoit Steiner; Ilya Sutskever; Kunal Talwar; Paul Tucker; Vincent Vanhoucke; Vijay Vasudevan; Fernanda Viégas; Oriol Vinyals; Pete Warden; Martin Wattenberg; Martin Wicke; Yuan Yu; Xiaoqiang Zheng. TensorFlow: Large-Scale Machine Learning on Heterogeneous Systems; 2015.

(19) Kingma, D. P.; Ba, J. Adam: A Method for Stochastic Optimization. arXiv preprint arXiv:1412.6980 2014.

(20) Feinberg, E. N.; Sur, D.; Wu, Z.; Husic, B. E.; Mai, H.; Li, Y.; Sun, S.; Yang, J.; Ramsundar, B.; Pande, V. S. PotentialNet for Molecular Property Prediction. ACS central science 2018, 4, 1520-1530.

(21) Morgan, H. L. The Generation of a Unique Machine Description for Chemical Structures-A Technique Developed at Chemical Abstracts Service. J. Chem. Doc. 1965, 5, 107-113. https://doi.org/10.1021/c160017a018.

(22) Rogers, D.; Hahn, M. Extended-Connectivity Fingerprints. Journal of chemical information and modeling 2010, 50, 742-754.

(23) Pedregosa, F.; Varoquaux, G.; Gramfort, A.; Michel, V.; Thirion, B.; Grisel, O.; Blondel, M.; Prettenhofer, P.; Weiss, R.; Dubourg, V. Scikit-Learn: Machine Learning in Python. Journal of machine learning research 2011, 12, 2825-2830.

(24) Dickson, C. J.; Madej, B. D.; Skjevik, A. A.; Betz, R. M.; Teigen, K.; Gould, I. R.; Walker, R. C. Lipid14: The Amber Lipid Force Field. J Chem Theory Comput 2014, 10, 865-879. https://doi.org/10.1021/ct4010307.

(25) Parrinello, M.; Rahman, A. Polymorphic Transitions in Single Crystals: A New Molecular Dynamics Method. Journal of Applied physics 1981, 52, 7182-7190. 